\title{
Correction to: Comprehensive benchmarking and ensemble approaches for metagenomic classifiers
}

\author{
Alexa B. R. Mclntyre ${ }^{1,2,3}$, Rachid Ounit ${ }^{4}$, Ebrahim Afshinnekoo ${ }^{2,3,5}$, Robert J. Prill ${ }^{6}$, Elizabeth Hénaff ${ }^{2,3}$, \\ Noah Alexander, ${ }^{2,3}$, Samuel S. Minot ${ }^{7}$, David Danko ${ }^{1,2,3}$, Jonathan Foox ${ }^{2,3}$, Sofia Ahsanuddin ${ }^{2,3}$, Scott Tighe ${ }^{8}$, \\ Nur A. Hasan ${ }^{9,10}$, Poorani Subramanian ${ }^{9}$, Kelly Moffat ${ }^{9}$, Shawn Levy ${ }^{11}$, Stefano Lonardi ${ }^{4}$, Nick Greenfield ${ }^{7}$, \\ Rita R. Colwell ${ }^{9,12}$, Gail L. Rosen ${ }^{13^{*}}$ and Christopher E. Mason ${ }^{2,3,14^{*}}$
}

\section{Correction to: Genome Biol (2017) 18:182 https://doi.org/10.1186/s13059-017-1299-7}

Following publication of the original article [1], the authors would like to highlight the following two corrections:

1) The updated "Availability of data and materials" declaration to the article:

The datasets and scripts supporting the conclusions of this article are freely and publicly available through the IMMSA server, ftp://ftpprivate.ncbi.nlm.nih.gov/nist-immsa/IMMSA/ Scripts used for analysis and generating figures are available at: https://scu.med.cornell.edu/git/ abm237/benchmarking_metagenomic_classifiers

2) The authors would like to clarify that the kraken $\mathrm{db}$ build command in the manuscript is for the bacterial database; the command for the standard $\mathrm{db}$ is available through the kraken manual: krakenbuild --standard --db \$DBNAME

\footnotetext{
Author details

${ }^{1}$ Tri-Institutional Program in Computational Biology and Medicine, New York, NY, USA. ${ }^{2}$ Department of Physiology and Biophysics, Weill Cornell Medicine, New York, NY 10021, USA. ${ }^{3}$ The HRH Prince Alwaleed Bin Talal Bin Abdulaziz Alsaud Institute for Computational Biomedicine, New York, NY 10021, USA. ${ }^{4}$ Department of Computer Science and Engineering, University of California, Riverside, CA 92521, USA. ${ }^{5}$ School of Medicine, New York Medical College, Valhalla, NY 10595, USA. ${ }^{6}$ Accelerated Discovery Lab, IBM Almaden Research Center, San Jose, CA 95120, USA. 'One Codex, Reference Genomics, San Francisco, CA 94103, USA. ${ }^{8}$ University of Vermont, Burlington, VT 05405, USA. ${ }^{9}$ CosmosID, Inc, Rockville, MD 20850, USA. ${ }^{10}$ Center for Bioinformatics and
}

Computational Biology, University of Maryland Institute for Advanced Computer Studies (UMIACS), College Park, MD 20742, USA. ${ }^{11}$ HudsonAlpha Institute for Biotechnology, Huntsville, AL 35806, USA. ${ }^{12}$ Johns Hopkins University Bloomberg School of Public Health, Baltimore, MD, USA.

${ }^{13}$ Department of Electrical and Computer Engineering, Drexel University, Philadelphia, PA 19104, USA. ${ }^{14}$ The Feil Family Brain and Mind Research Institute, New York, NY 10065, USA.

Received: 1 April 2019 Accepted: 1 April 2019

Published online: 05 April 2019

\section{Reference}

1. Mclntyre $A B R$, et al. Comprehensive benchmarking and ensemble approaches for metagenomic classifiers. Genome Biol. 2017;18:182 https://doi.org/10.1186/s13059-017-1299-7.

*Correspondence: gail.I.rosen@gmail.com; chm2042@med.cornell.edu

${ }^{13}$ Department of Electrical and Computer Engineering, Drexel University, Philadelphia, PA 19104, USA

${ }^{2}$ Department of Physiology and Biophysics, Weill Cornell Medicine, New York, NY 10021, USA

Full list of author information is available at the end of the article

(c) The Author(s). 2019 Open Access This article is distributed under the terms of the Creative Commons Attribution 4.0 International License (http://creativecommons.org/licenses/by/4.0/), which permits unrestricted use, distribution, and reproduction in any medium, provided you give appropriate credit to the original author(s) and the source, provide a link to the Creative Commons license, and indicate if changes were made. The Creative Commons Public Domain Dedication waiver (http://creativecommons.org/publicdomain/zero/1.0/) applies to the data made available in this article, unless otherwise stated. 